\title{
Seeking Opportunities in Adverse Scenarios: A Challenge for Psychology
}

We have closed off yet another year involved in the countless tasks that are part of the editorial process of a scientific journals committed to continuing improvement. The manuscripts are fully published in English, so as to guarantee the journal's international insertion. We hope to continue attracting the best scientific contributions, which will reflect in a quality increase as a whole and in a higher impact factor.

We are convinced that conquering a place in the sun on the international Psychology scene involves the efforts of different generations and the perpetual commitment to the targets set through a coordinated action plan established by each journal's Editorial Board. Much remains to be done in the editorial management of Brazilian Psychology journal, in terms of editorial quality, dissemination, standardization, review process and acknowledgement of authorship, discussing the strengths and weaknesses, the advantages of open access to scientific information, the fundamental need to further value and qualify the work of editors and consultants. Particularly complex issues need to be included on the agenda, such as the need for funding to sustain the journals, which does not necessarily derive from submission and/or publication fees. And, above all, there is the urgent challenge to professionalize the editorial teams of Psychology journals.

This issue 59, volume 24 of Paidéia opens with the article entitled Interparental Conflicts and the Development of Psychopathology in Adolescents and Young Adults, authored by Olga Melo and Catarina Pinheiro Mota, from Universidade de Trás-os-Montes e Alto Douro, Portugal, which analyzed to what extent inter parental conflicts and divorce act as predictors of psychopathological development in young people from intact and divorced families. The participants were 827 Portuguese young people between 13 and 25 years of age. The children whose parents are separated or divorced perceive the frequency and intensity of inter parental conflicts more highly, but present a higher solution level of the inter parental conflicts and higher psychopathology levels. Nevertheless, the family structure has no moderating effect on the interaction between the inter parental conflicts and the development of psychopathology.

Next, the article Life Satisfaction in Women With Breast Cancer, by Solange Fonseca, Leonor Lencastre, and Marina Guerra, from Universidade do Porto, Portugal, aimed to analyze the relationship between satisfaction with life, meaning of life, optimism, body image and depression in 55 women with breast cancer clustered within two groups: mastectomized and submitted to conservative surgery. Meaning of life and optimism had positive correlation to satisfaction with life. Higher depression and body image disturbance appeared associated with minor satisfaction with life. Body image disturbance was higher in mastectomized women. It is concluded that women submitted to conservative surgery are not more satisfied with life than mastectomized ones, needing equal attention regarding depression prevention and positive variables promotion.

Following, the article The Positivity Dimension of Well-Being: Adaptation and Psychometric Evidence of a Measure, by Roosevelt Vilar Lobo de Souza, Rafaella de Carvalho Rodrigues Araújo, and Valdiney Veloso Gouveia, from Universidade Federal da Paraiba, Brazil, Rildésia Silva Veloso Gouveia, from Centro Universitário João Pessoa, Brazil, and Gabriel Lins de Holanda Coelho, from Cardiff University, Wales, aimed to adapt the Positivity Scale (PS) to the Brazilian context, gathering evidence of validity and reliability. Two studies were performed. Study 1 was composed by 200 people from Paraíba, who answered the PS and demographic questions. Results pointed out to a one-factor solution in this scale, which presented satisfactory reliability. Study 2 gathered 290 undergraduate students, who answered the PS, the Satisfaction with Life Scale, the Subjective Vitality Scale and demographic questions. Confirmatory factor analyses (ML and ADF estimators) corroborated the one-factor structure, which presented an acceptable reliability. Furthermore, its convergent validity was confirmed based on the average extracted variance and on its correlations with satisfaction with life and vitality. In conclusion, this measure has shown to be psychometrically adequate for being used in Brazil.

The study Emerging Adulthood in Brazilians of Differing Socioeconomic Status: Transition to Adulthood, written by Luciana Dutra-Thomé and Silvia Helena Koller, from Universidade Federal do Rio Grande do Sul, Brazil, investigated into the transition to adulthood in Brazilian young people of differing socioeconomic status (SES), considering the phenomenon of Emerging Adulthood (EA). The variables analyzed were sociodemographic aspects, perceptions of adulthood, access to technology, and educational and employment status. The sample included 547 young people and more than $50 \%$ of the total sample reported that they felt in-between adolescence and adulthood, which may indicate the existence of EA in individuals of both SES. However, EA was more likely to be present in high SES contexts. The low SES group presented a tendency to assume adult responsibilities earlier, which blocks their opportunities for experiencing a period of identity exploration.

Subsequently, the article Self-Regulated Learning in Students of Pedagogy, authored by Janete Aparecida da Silva Marini, from Faculdades Padre Anchieta, Brazil, and Evely Boruchovitch, from Universidade Estadual de Campinas, Brazil, investigated relationships between motivation to learn, implicit theories of intelligence and self-handicapping strategies, and to examine the association 
of these variables in the prediction of the use of learning strategies in students of Pedagogy. The sample consisted of 107 Pedagogy students of two private universities of a city of São Paulo state. Multivariate linear regression analyses revealed that participants with higher scores in the learning strategies scale also presented significantly higher scores in intrinsic motivation and fewer reports of the use of selfhandicapping strategies. Higher scores in metacognitive strategies were significantly associated with both intrinsic an extrinsic motivation and with fewer reports of the use of selfhandicapping strategies.

The study Mental Health, Mother-Child Interaction and Development at the End of the First Year of Life, by Débora Gerardo Ribeiro, Gimol Benzaquen Perosa, and Flávia Helena Pereira Padovani, from Universidade Estadual Paulista “Júlio de Mesquita Filho", Brazil, aimed to evaluate the relationship between the mother-child interaction and the neuro-psychomotor development of children at one year of age, taking into consideration the maternal mental health. A total of 65 children participated, along with their mothers, who responded to a screening interview for common mental disorders. Developmental risk was presented by $43.1 \%$ of the children. Although $44.6 \%$ of the mothers presented scores indicative of psychological distress, maternal mental health was not associated with the interactive behaviors. Some maternal and child behaviors were associated with developmental delays, however, only cognitive stimulation was shown to be a predictor of delays.

The article Beliefs of Mothers, Nannies, Grandmothers and Daycare Providers Concerning Childcare, authored by Maria Lucia Seidl-de-Moura, from Universidade do Estado do Rio de Janeiro, Brazil, Luciana Fontes Pessôa, from Pontifícia Universidade Católica do Rio de Janeiro, Brazil, Dandara de Oliveira Ramos and Deise Maria Leal Fernandes Mendes, from Universidade do Estado do Rio de Janeiro, Brazil, Ana Carolina Monnerat Fioravanti-Bastos, from Universidade Federal Fluminense, Brazil, and Luciana Brooking, from Universidade do Estado do Rio de Janeiro, Brazil, analyzed potential differences among distinct profiles of caregivers regarding the valuation of Keller's parenting systems. A total of 120 caregivers of children younger than one year of age participated in a semi-structured interview. Significant differences were found among the caregivers in regard to the importance they assigned to the different systems: face-to-face, body stimulation and basic care. Education also significantly influenced the valorization of face-to-face and basic care systems. The conclusion is that the caregivers presented a mixed parental style, both distal, enabling the experience of autonomy and separation, and proximal, valuing greater interpersonal relationships.

The upcoming article, Pain and Distress in Inpatient Children According to Child and Mother Perceptions, written by Ana Claudia Matsuda-Castro and Maria Beatriz Martins Linhares, from Universidade de São Paulo, Brazil, examined the associations between pain experiences in children with respect to self-perceptions and mother perceptions and stress indicators. The study sample included 30 hospitalized children of 6-12 years of age and their mothers. The results showed that higher pain intensities in children was associated with higher stress, psychological and psychophysiological reactions in children. Higher pain intensities in children also increase mothers' perceptions of pain in children. Seventy percent of the mothers presented clinical indicators of stress. Psychological support during child hospitalization should address the management of pain and stress in children and stress in mothers.

The study entitled Creativity and Intelligence in Children With and Without Developmental Dyslexia, by Rauni Jandé Roama Alves and Tatiana de Cássia Nakano, from Pontificia Universidade Católica de Campinas, Brazil, verified if the creativity of children with Developmental Dyslexia (CG, $n=$ 13) differs from children without difficulties in reading and writing (NG, $n=13$ ), and how creativity could be related to intelligence. High correlations were found between intelligence and creativity in both groups, and variable correlations for the same type of analysis were found in the literature. There were no significant intergroup differences for creativity, although the NG had a higher overall average in creativity and the $\mathrm{CG}$, the highest averages in most evaluated creative characteristics.

The next article, Linguistic Repertoires of Interdisciplinarity in Brazilian Journals in the Area of Psychology, written by Mary Jane Paris Spink, from Pontifícia Universidade Católica de São Paulo, Brazil, Marcos Antonio dos Santos Reigota, from Universidade de Sorocaba, Brazil, and Mário Henrique da Mata Martins, from Pontificia Universidade Católica de São Paulo, Brazil, investigated the manners in which linguistic repertoires of interdisciplinarity for dissemination of scientific knowledge are coordinated. Also investigated how ordering, controlling and coordinating interdisciplinary operates in the dissemination of scientific knowledge. The analysis of the ways of coordinating interdisciplinarity in scientific dissemination was based on the editorial proposals of journals classified as A1, A2 and B1 in the Qualis of the area of Psychology. The conclusion of this analysis is that scientific journals enact interdisciplinarity in different manners because they use various forms of association based on themes, related areas, and theories or theoretical frameworks.

Following the contributions, the article Subjective Well-Being and Time Use of Brazilian PhD Professors, by Maiana Farias Oliveira Nunes, from Faculdade Avantis, Brazil, Claudio Simon Hutz, from Universidade Federal do Rio Grande Do Sul, Brazil, Jeferson Gervasio Pires and Cassandra Melo Oliveira, from Universidade Federal de Santa Catarina, Brazil, aimed to analyze the subjective well-being, strategies for time use and satisfaction with time use in Ph.D.s teachers both with and without productivity scholarship by CNPq. Participants were 83 teachers, mean 
age 50 years, $89 \%$ from state institutions. Most participants presented high level of negative affect and low level of satisfaction with time use. The most relevant reason for dissatisfaction with time use was excess of work, whereas peer recognition was the main achievement obtained with work. The article discusses how demands and work conditions affect the well-being of these.

The study entitled Alterity and Identity Refusal: The Construction of the Image of the Crack User, authored by Manoel de Lima Acioli Neto and Maria de Fátima de Souza Santos, from Universidade Federal de Pernambuco, Brazil, addressed the construction of otherness around the image of crack users. Fourteen crack users were interviewed in different locations and the results suggest that the image of crack users is established on an alterity. Thus, despite the users realized that their actions is not determined from the standards from their interactional networks, hegemonic representations of their use contexts refer these activities as truths about the crack. Thereby, even experiencing other experiences with the drug, they believe that its use refers to the scope of the destructive pleasure and the impossibility of voluntary action.

Concluding this issue, the review entitled Analysis of Studies on Social Support and Children of Depressed Mothers: A Systematic Review, written by Ana Paula Casagrande Silva and Sonia Regina Loureiro, from Universidade de São Paulo, Brazil, aimed to analyze recent empirical studies that have investigated the associations between maternal depression and different social support conditions for school-aged children. This review included and analyzed twenty-seven empirical articles indexed from 2007 to 2014 in various databases. The selected studies included a broad range of contextual risk factors and social support conditions, and in their predictive models, it was found that less contextual risk and greater parental resources and social support were predictors of fewer problems for the children. The identification of social support sources that attenuate the effect of maternal depression on child outcomes has implications for the development of prevention and intervention programs.

This issue closes with the Paidéia Publication Standards. We hope the readers can appreciate the richness of the contributions and have a fruitful reading.

Manoel Antônio dos Santos

Editor

How to cite this editorial:

Santos, M. A. (2014). Seeking opportunities in adverse scenarios: A challenge for Psychology. [Editorial]. Paidéia (Ribeirão Preto), 24(59), 279-281. doi: 10.1590/1982-43272459201401 


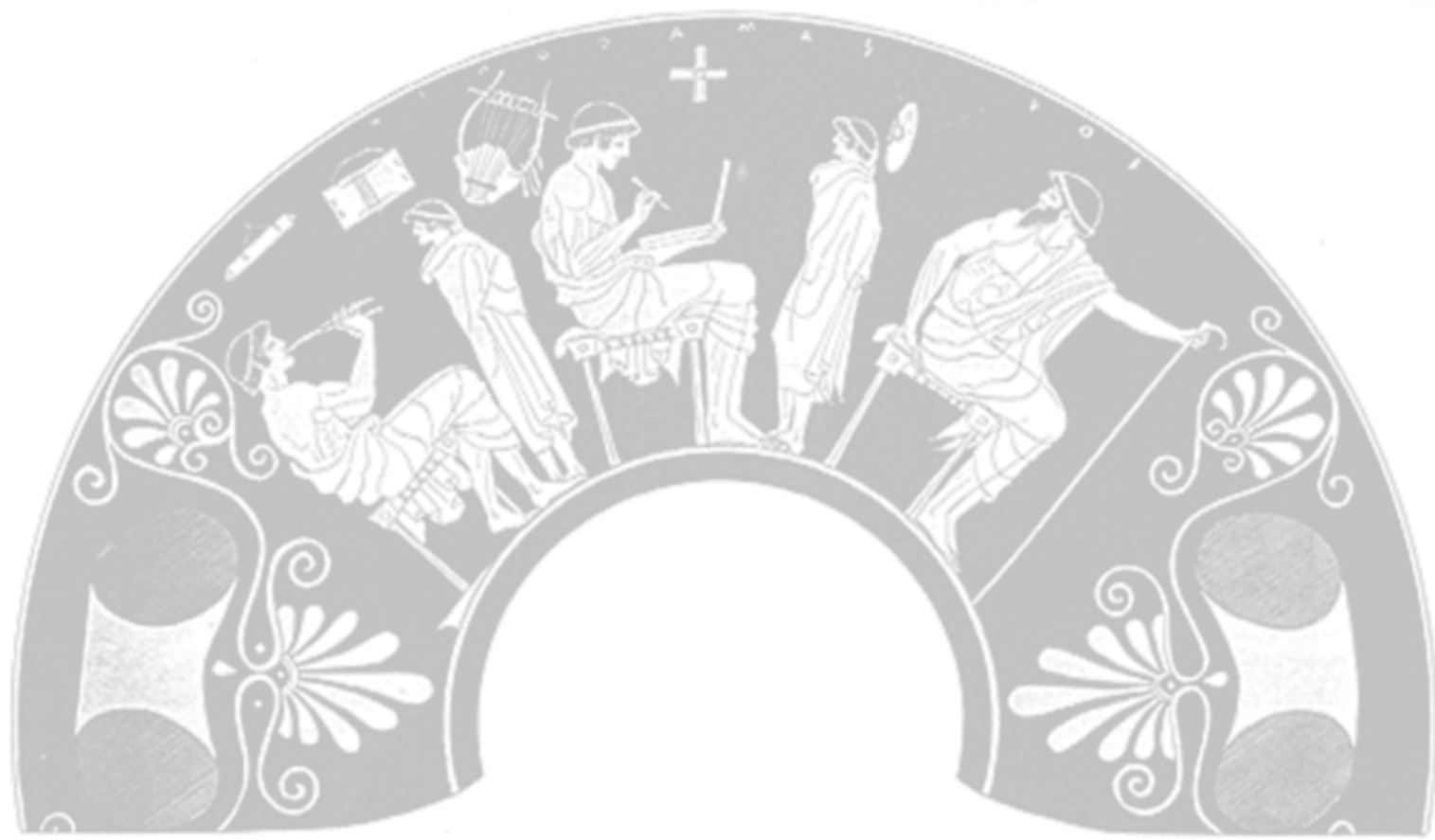

